
Not for reproduction, distribution or commercial use.

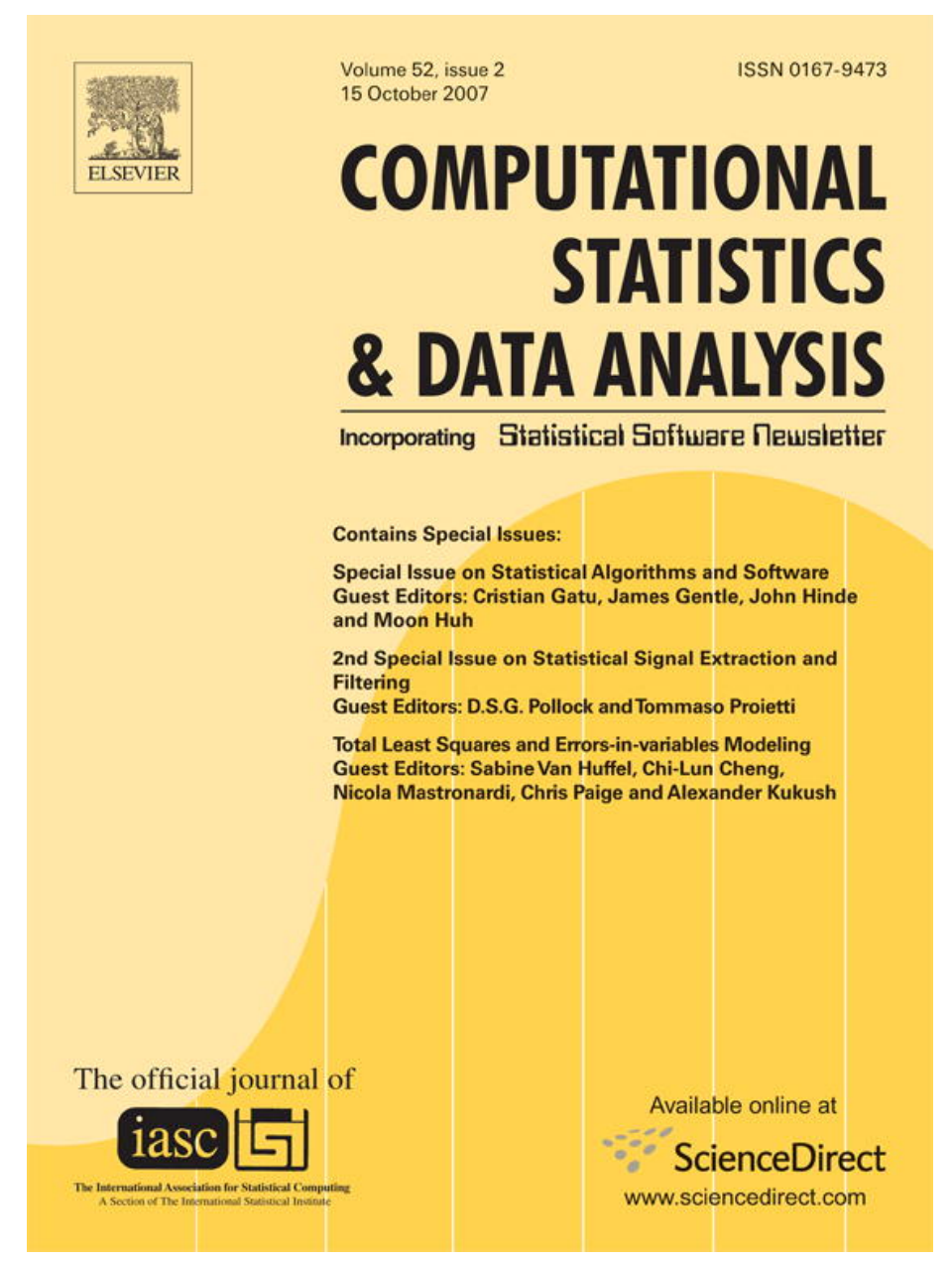

This article was published in an Elsevier journal. The attached copy

is furnished to the author for non-commercial research and education use, including for instruction at the author's institution, sharing with colleagues and providing to institution administration.

Other uses, including reproduction and distribution, or selling or licensing copies, or posting to personal, institutional or third party websites are prohibited.

In most cases authors are permitted to post their version of the article (e.g. in Word or Tex form) to their personal website or institutional repository. Authors requiring further information regarding Elsevier's archiving and manuscript policies are encouraged to visit: 


\title{
A unifying framework for analysing common cyclical features in cointegrated time series
}

\author{
Gianluca Cubadda* \\ Dipartimento SEFEMEQ, Universita' di Roma “Tor Vergata” Via Columbia 2, 00133 Roma, Italy
}

Available online 14 July 2007

\begin{abstract}
A unifying framework in which the coexistence of differing forms of common cyclical features can be tested and imposed upon a cointegrated VAR model is provided. This is achieved by introducing a new notion of common cyclical features, described as the weak form of polynomial serial correlation, which encompasses most of the existing formulations. Statistical inference is based upon reduced-rank regression, and alternative forms of common cyclical features are detected through tests for over-identifying restrictions on the parameters of the new model. Some iterative estimation procedures are then proposed for simultaneously modelling various forms of common features. The concepts and methods of the paper are illustrated via an empirical investigation of the US business cycle indicators.
\end{abstract}

(C) 2007 Elsevier B.V. All rights reserved.

Keywords: Common features; Reduced rank regression

\section{Introduction}

Many of the recent advances in modelling multiple time series have been concerned with the analysis of comovements amongst economic variables. A common concept of long-run comovements is cointegration, according to which a vector of I(1) time series is cointegrated when its elements share some common stochastic trends (Engle and Granger, 1987). However, detrended economic variables often display quite similar cyclical patterns (Lucas, 1977). This well-known "stylized fact" suggests that economic time series will tend to share common transitory components as well. Engle and Kozicki (1993) proposed the notion of common serial correlation feature to detect short-run comovements among I(1) variables. Indeed, common cycles exist in the multivariate Beveridge and Nelson (1981) decomposition of a multiple I(1) time series when its first differences exhibit common serial correlation (Vahid and Engle, 1993). The idea of common cycles has later been extended to seasonally integrated series (Cubadda, 1999), I(2) systems (Paruolo, 2006a), and periodically integrated series (Haldrup et al., 2007).

From the statistical viewpoint, the presence of common cycles allows the vector error correction model (VECM) to be reformulated as a reduced-rank regression (RRR) model. This implies that RRR techniques (see, inter alia, Johansen, 1996; Reinsel and Velu, 1998) can be used to obtain a more parsimonious model of the data. However, this notion of common cycles is somewhat limited since it does not enable one to detect the presence of non-synchronous cycles among I(1) time series (Ericsson, 1993). Consequently, some variants of the common cycles model have previously

\footnotetext{
* Tel.: +390672595847; fax: +39062040219.

E-mail address: gianluca.cubadda@uniroma2.it.
} 
been proposed in order to overcome this limitation. In this paper, attention is focussed on the forms of common serial correlation that lead to a VECM with a partial reduced-rank structure. Such forms are the polynomial common features that have been described by Cubadda and Hecq (2001) and the weak form of common serial correlation that has been described by Hecq et al. (2000, 2006).

A serious limitation of existing methods for analysing common features is that they cannot incorporate several forms of common serial correlation in the same VECM. Thus, although one can test for the presence of the various forms of common features, existing procedures do not allow one to impose the implied reduced-rank structures on the estimated model, and, therefore, the most parsimonious model cannot be fitted to the data.

The goal of this paper is threefold. First, it provides a new interpretation of the weak form of common serial correlation that has implications for the short-run components of the series. Second, it proposes a new notion, named weak form of polynomial common features, which encompasses all forms of common serial correlation that have hitherto been considered in this context. Third, it is shown how diverse forms of common features can be detected and imposed upon the estimated VECM. This goal is achieved by means of an iterative estimation procedure which is similar in the spirit to the method that was proposed by Cubadda and Omtzigt (2005) to estimate the cointegration vectors jointly at the zero and seasonal frequencies. In contrast to the nested reduced rank autoregressive model by Ahn and Reinsel (1988), the new procedure can be applied even when the coexisting forms of common features are not nested.

This paper is organized as follows. Section 1 reviews some forms of common serial correlation and it introduces the weak form of polynomial common features. Section 2 deals with the problem of simultaneously modelling differing forms of common features. In Section 3, the methodology is applied to some US business cycle indicators. Section 4 contains the conclusions.

\section{Alternative notions of common cyclical features}

Let us assume that an $n$-vector $\left\{y_{t}, t=1, \ldots, T\right\}$ of cointegrated time series of order $(1,1)$ is generated by the following VECM:

$$
\Gamma(L) \Delta y_{t}=\Phi_{0}+\alpha \beta_{*}^{\prime} y_{t-1}^{*}+\varepsilon_{t},
$$

for fixed values of $y_{-p+1}, \ldots, y_{0}$, where $\beta_{*}^{\prime}=\left(\beta^{\prime}, \Phi_{1}^{\prime}\right), \alpha$ and $\beta$ are both $(n \times r)$ matrices of full-rank $r, \Phi_{1}$ is an $r$-vector, $\Gamma(L)=I_{n}-\sum_{i=1}^{p-1} \Gamma_{i} L^{i}$ is such that the matrix $\alpha_{\perp}^{\prime} \Gamma(1) \beta_{\perp}$ has rank equal to $(n-r)$ and $\operatorname{det}\left(\Gamma(z)(1-z)-\alpha \beta^{\prime} z\right)=0$ implies that $z=1$ or $|z|>1, y_{t}^{*}=\left(y_{t}^{\prime}, t\right)^{\prime}$, and $\varepsilon_{t}$ are i.i.d. $N_{n}\left(0, \Sigma_{\varepsilon}\right)$ innovations if $t \geqslant 1$ and an $n$-vector of zeros otherwise.

Since $\Delta y_{t}$ is a stationary stochastic process, it admits the following Wold representation

$$
\Delta y_{t}=\mu+C(L) \varepsilon_{t},
$$

where $C(L)=I_{n}+\sum_{j=1}^{\infty} C_{j}$, the coefficient matrices $C_{j}$ decrease exponentially fast, and $\mu=\Phi_{0}+\alpha \beta^{\prime} y_{0}+$ $\sum_{i=1}^{p-1} \Gamma_{i} \Delta y_{1-i}$ (see e.g. Johansen, 1996). From the expansion

$$
C(L)=C(1)+\Delta C^{*}(L),
$$

where $C_{i}^{*}=-\sum_{i+1}^{\infty} C_{j}$ for $i \geqslant 0$, we obtain the multivariate BN representation (Beveridge and Nelson, 1981) of the series $y_{t}$

$$
y_{t}=\delta_{t}+\tau_{t}+\kappa_{t},
$$

where $\delta_{t}=y_{0}+\mu t, \tau_{t}=C(1) \sum_{i=0}^{t-1} \varepsilon_{t-i}$, and $\kappa_{t}=C^{*}(L) \varepsilon_{t}$. Based on the popular view that the stochastic trend of an I(1) time series is a random walk, the processes $\tau_{t}$ and $\kappa_{t}$ are, respectively, defined as the stochastic trends and cycles of variables $y_{t}$. Proietti (1997) discusses in details the relations among the multivariate BN representation and other popular permanent-transitory decompositions.

It is well known that the presence of cointegration is equivalent to the existence of $(n-r)$ common stochastic trends since $\beta^{\prime} \tau_{t}=0$ (Engle and Granger, 1987). Hence, a reduced rank restriction on the coefficient matrix of the terms $y_{t-1}$ in model (1) is associated with a reduced number of components that are responsible for the long-run behaviour of series $y_{t}$. 
The analysis of common cyclical features is instead concerned with the short-run components of series $y_{t}$. In particular, the focus is on additional reduced-rank restrictions on the parameters of model (1) that have interesting implications for the cycles $\kappa_{t}$. Let us briefly review the various forms of common cyclical features which have gained some attention in the literature, starting with the seminal notion of common cyclical features proposed by Engle and Kozicki (1993):

Definition 1 (Serial correlation common feature (SCCF)). Series $\Delta y_{t}$ have $s(s<n)$ SCCFs iff there exists an $n \times s$ matrix $\delta_{S}$ with full column rank such that the VECM in (1) can be rewritten as the following RRR model:

$$
\Delta y_{t}=\Phi_{0}+\delta_{S \perp} \psi_{S}^{\prime} w_{t-1}+\varepsilon_{t},
$$

where for any full column rank matrix $M$ we denote by $M_{\perp}$ a full column rank matrix such that $M^{\prime} M_{\perp}=0, \psi_{S}$ is an $(n p-n+r) \times(n-s)$ matrix with full column rank, and $w_{t-1}=\left(y_{t-1}^{* \prime} \beta_{*}, \Delta y_{t-1}^{\prime}, \ldots, \Delta y_{t-p+1}^{\prime}\right)^{\prime}$.

The distinctive property of model (4) is that the predictable variations of series $\Delta y_{t}$ are entirely generated by the $(n-s)$ common factors $\psi_{S}^{\prime} w_{t-1}$. Indeed, by premultiplying both sides of Eq. (4) by $\delta_{S}^{\prime}$ it follows that

$$
\delta_{S}^{\prime} \Delta y_{t}=\delta_{S}^{\prime} \Phi_{0}+\delta_{S}^{\prime} \varepsilon_{t} .
$$

Hence, the SCCF requires that there exists a linear combination of series $\Delta y_{t}$ that is an innovation with respect to $\Omega_{t-1}$, where $\Omega_{t}$ is the $\sigma$-field generated by $\left\{y_{t-i} ; i \geqslant 0\right\}$. Moreover, the presence of $s$ SCCFs is equivalent to the existence of $(n-s)$ common cycles since, as shown by Vahid and Engle (1993), $\delta_{S}^{\prime} \kappa_{t}=0$.

Remark 1. Another well-known notion of common autocorrelation is discussed in the so-called common factor analysis, see, inter alia, Sargan (1983) and Mizon (1995). However, it is easy to check there is no relation of implication between these two notions. A proof is available upon request.

A drawback of the above definition is that it does not take account of the possibility that common serial correlation is present among non-contemporaneous elements of series $\Delta y_{t}$ (see, e.g., Ericsson, 1993). In order to overcome this limitation, Cubadda and Hecq (2001) introduced the following variant of the SCCF.

Definition 2 (Polynomial serial correlation common feature (PSCCF)). Series $\Delta y_{t}$ have $s$ PSCCFs iff there exists an $n \times s$ matrix $\delta_{P}$ with full column rank such that $\delta_{P}^{\prime} \Gamma_{1} \neq 0$, and the VECM in (1) can be rewritten as the following partial RRR model:

$$
\Delta y_{t}=\Phi_{0}+\Gamma_{1} \Delta y_{t-1}+\delta_{P \perp} \psi_{P}^{\prime}\left(\Delta y_{t-2}^{\prime}, \ldots, \Delta y_{t-p+1}^{\prime}, y_{t-1}^{* \prime} \beta_{*}\right)^{\prime}+\varepsilon_{t},
$$

where $\psi_{P}$ is an $(n p-2 n+r) \times(n-s)$ matrix with full column rank.

In order to interpret the notion of PSCCF, let us premultiply both sides of Eq. (5) by $\delta_{P}^{\prime}$. We then obtain

$$
\delta(L)^{\prime} \Delta y_{t}=\delta_{P}^{\prime} \Phi_{0}+\delta_{P}^{\prime} \varepsilon_{t},
$$

where $\delta(L)=\delta_{P}-\Gamma_{1}^{\prime} \delta_{P} L$. Hence, the PSCCF requires that there exists a first-order polynomial matrix $\delta(L)$ such that $\delta(L)^{\prime} \Delta y_{t}$ is unpredictable from the past. Notice that the notion of PSCCF can be easily generalized to the case where the polynomial matrix $\delta(L)$ is of order $m$, where $m \leqslant(p-1)$. See Cubadda and Hecq (2001) for details.

The existence of the PSCCF has an interesting implication for the BN cycles of series $y_{t}$. Indeed, Cubadda and Hecq (2001) proved that $\delta(L)^{\prime} \kappa_{t}=-\delta_{P}^{\prime} \Gamma_{1} C(1) \varepsilon_{t}$. Hence, the same PSCCF relationships cancel the dependence from the past of both the first differences and cycles of series $y_{t}$.

Notice that Eqs. (4) and (5) imply that both the matrices $\delta_{S}$ and $\delta_{P}$ must lie in the left-null space of the error-correction term loading matrix $\alpha$. Hence, the number of the SCCFs or PSCCFs, $s$, cannot exceed the number of common trends $(n-r)$. In order to remove this restriction, Hecq et al. $(2000,2006)$ proposed the following notion of weak form of SCCF. 
Definition 3 (Weak form of serial correlation common feature (WF)). Series $\Delta y_{t}$ have $s$ WFs iff there exists an $n \times s$ matrix $\delta_{W}$ with full column rank such that $\delta_{W}^{\prime} \alpha \neq 0$, and the VECM in (1) can be rewritten as the following partial RRR model:

$$
\Delta y_{t}=\Phi_{0}+\alpha \beta_{*}^{\prime} y_{t-1}^{*}+\delta_{W \perp} \psi_{W}^{\prime}\left(\Delta y_{t-1}^{\prime}, \ldots, \Delta y_{t-p+1}^{\prime}\right)^{\prime}+\varepsilon_{t},
$$

where $\psi_{W}$ is an $(n p-n) \times(n-s)$ matrix with full column rank.

The usual interpretation of the WF is that there exists a linear combination of series $\left(\Delta y_{t}-\alpha \beta_{*}^{\prime} y_{t-1}^{*}\right)$ that is an innovation with respect to $\Omega_{t-1}$. It is, however, possible to provide a new reading that permits one to uncover an interesting implication of the WF for the BN cycles $\kappa_{t}$. Indeed, premultiplying both sides of Eq. (6) by $\delta_{W}^{\prime}$ yields

$$
\delta_{W}(L)^{\prime} y_{t}=\delta_{W}^{\prime}\left(\Phi_{0}+\alpha \Phi_{1}^{\prime} t\right)+\delta_{W}^{\prime} \varepsilon_{t},
$$

where $\delta_{W}(L)=\delta_{W}-\left(\beta \alpha^{\prime}+I_{n}\right) \delta_{W} L$. By substituting (3) into (2) and premultiplying both sides of the resulting equation by $\delta_{W}(L)^{\prime}$ one obtains

$$
\delta_{W}(L)^{\prime} \Delta y_{t}=\delta_{W}(1)^{\prime} \mu+\delta_{W}(L)^{\prime}\left[C(1)+\Delta C^{*}(L)\right] \varepsilon_{t} .
$$

Finally, by taking first differences of both sides of (7) and comparing the resulting equation with the one above, it follows that

$$
\delta_{W}(L)^{\prime} \kappa_{t} \equiv \delta_{W}(L)^{\prime} C^{*}(L) \varepsilon_{t}=\delta_{W}^{\prime}\left(I_{n}-C(1)\right) \varepsilon_{t} .
$$

The above results, which highlight that the WF is an analogous property to the PSCCF that applies to the levels rather than to the differences of series $y_{t}$, are summarized in the following proposition:

Proposition 1. Series $\Delta y_{t}$ have $s$ WFs iff there exists a first-order polynomial matrix $\delta_{W}(L)$ such that $\left(\delta_{W}^{\prime}(L) y_{t}-\right.$ $\left.\alpha \delta_{W}^{\prime} \Phi_{1}^{\prime} t\right)$ is an innovation process with respect to $\Omega_{t-1}$. Moreover, $\delta_{W}(L)^{\prime} \kappa_{t}$ is also an innovation.

Remark 2. As correctly pointed out by a referee, the original definition of WF is not invariant to reparametrizations of the VECM such as the one where the EC terms appear as $\beta^{\prime} y_{t-p}$ in place of $\beta^{\prime} y_{t-1}$. However, it is easy to see that the definition of WF in terms of the polynomial matrix $\delta_{W}(L)$ does not suffer from this non-uniqueness problem. A proof is available upon request.

Interestingly enough, it is possible to merge the notions of PSCCF and WF as follows.

Definition 4 (Weak form of polynomial serial correlation common feature (WFP)). Series $\Delta y_{t}$ have $s$ WFPs iff there exists an $n \times s$ matrix $\delta_{F}$ with full column rank such that $\delta_{F}^{\prime} \alpha \neq 0, \delta_{F}^{\prime} \Gamma_{1} \neq 0$, and the VECM in (1) can be rewritten as the following partial RRR model:

$$
\Delta y_{t}=\Phi_{0}+\alpha \beta_{*}^{\prime} y_{t-1}^{*}+\Gamma_{1} \Delta y_{t-1}+\delta_{F \perp} \psi_{F}^{\prime}\left(\Delta y_{t-2}^{\prime}, \ldots, \Delta y_{t-p+1}^{\prime}\right)^{\prime}+\varepsilon_{t},
$$

where $\psi_{F}$ is an $(n p-2 n) \times(n-s)$ matrix with full column rank.

By premultiplying both sides of Eq. (8) by $\delta_{F}^{\prime}$ we see that the WFP requires the existence of a second-order polynomial matrix $\delta_{F}(L)=\delta_{F}-\left(\beta \alpha^{\prime}+I_{n}+\Gamma_{1}^{\prime}\right) \delta_{F} L+\Gamma_{1}^{\prime} \delta_{F} L^{2}$ such that

$$
\delta_{F}(L)^{\prime} y_{t}=\delta_{F}^{\prime}\left(\Phi_{0}+\alpha \Phi_{1}^{\prime} t\right)+\delta_{F}^{\prime} \varepsilon_{t} .
$$

In order to establish the implications of the WFP for the cycles $\kappa_{t}$, let us substitute (3) into (2) and premultiply both sides of the resulting equation by $\delta_{F}(L)^{\prime}$. We obtain that

$$
\delta_{F}(L)^{\prime} \Delta y_{t}=\delta_{F}(1)^{\prime} \mu+\delta_{F}(L)^{\prime}\left[C(1)+\Delta C^{*}(L)\right] \varepsilon_{t} .
$$

Finally, by taking first differences of both sides of (9) and in view of the above equation one obtains

$$
\delta_{F}(L)^{\prime} \kappa_{t} \equiv \delta_{F}(L)^{\prime} C^{*}(L) \varepsilon_{t}=\delta_{F}^{\prime}\left[I_{n}-C(1)\right] \varepsilon_{t}-\delta_{F}^{\prime} \Gamma_{1} C(1) \varepsilon_{t-1} .
$$


Table 1

Canonical correlations and tests for common features

\begin{tabular}{|c|c|c|c|}
\hline Model & $x_{t}$ & $z_{t}$ & $d_{1}$ \\
\hline (4) & $\left(\Delta y_{t-1}^{\prime}, \ldots, \Delta y_{t-p+1}^{\prime}, y_{t-1}^{* \prime} \beta_{*}\right)^{\prime}$ & 1 & $s \times(n(p-2)+r+s)$ \\
\hline (6) & $\left(\Delta y_{t-1}^{\prime}, \ldots, \Delta y_{t-p+1}^{\prime}\right)^{\prime}$ & $\left(1, y_{t-1}^{* \prime} \beta_{*}\right)^{\prime}$ & $s \times(n(p-2)+s)$ \\
\hline (5) & $\left(\Delta y_{t-2}^{\prime}, \ldots, \Delta y_{t-p+1}^{\prime}, y_{t-1}^{* \prime} \beta_{*}\right)^{\prime}$ & $\left(1, \Delta y_{t-1}^{\prime}\right)^{\prime}$ & $s \times(n(p-3)+r+s)$ \\
\hline (8) & $\left(\Delta y_{t-2}^{\prime}, \ldots, \Delta y_{t-p+1}^{\prime}\right)^{\prime}$ & $\left(1, \Delta y_{t-1}^{\prime}, y_{t-1}^{* \prime} \beta_{*}\right)^{\prime}$ & $s \times(n(p-3)+s)$ \\
\hline
\end{tabular}

Table 2

Estimators of the common features vectors and RRR coefficients

\begin{tabular}{lll}
\hline Model & $\left(\widehat{\varphi}_{1}^{\Delta y}, \ldots, \widehat{\varphi}_{s}^{\Delta y}\right)$ & $\left(\widehat{\varphi}_{s+1}^{x}, \ldots, \widehat{\varphi}_{n}^{x}\right)$ \\
\hline$(4)$ & $\widehat{\delta}_{S}$ & $\widehat{\psi}_{S}$ \\
$(6)$ & $\widehat{\delta}_{W}$ & $\widehat{\psi}_{W}$ \\
$(5)$ & $\widehat{\delta}_{P}$ & $\widehat{\psi}_{P}$ \\
$(8)$ & $\widehat{\delta}_{F}$ & \\
\hline
\end{tabular}

Hence, the second-order polynomial matrix $\delta_{F}(L)$ transforms the BN cycles $\kappa_{t}$ into a VMA(1) process.

Let $\operatorname{Can} \operatorname{Cor}\left\{\Delta y_{t}, x_{t} \mid z_{t}\right\}$ denote the partial canonical correlations between series $\Delta y_{t}$ and $x_{t}$ having removed the linear dependence on $z_{t}$. Maximum likelihood (ML) inference on the various forms of common features is obtained by solving CanCor $\left\{\Delta y_{t}, x_{t} \mid z_{t}\right\}$ for proper choices of the variables $x_{t}$ and $z_{t}$. In particular, let $\widehat{\lambda}_{i}$ denotes the $i$ th smallest squared partial canonical correlation for $i=1, \ldots, n$. Under the null that $s$ common features of a given form exist, the test statistic

$$
L R_{1}=-T \sum_{i=1}^{s} \ln \left(1-\widehat{\lambda}_{i}\right), \quad s=1, \ldots, n
$$

is asymptotically distributed as a $\chi^{2}\left(d_{1}\right)$ as detailed in Table 1, see, inter alia, Anderson (2002) and Paruolo (2003).

Moreover, let $\widehat{\varphi}_{i}^{\Delta y}$ and $\widehat{\varphi}_{i}^{x}$, respectively, denote the partial canonical coefficients of $\Delta y_{t}$ and $x_{t}$ associated with $\widehat{\lambda}_{i}$. Optimal estimates of both the common features vectors and (partial) RRR coefficients are then obtained as described in Table 2.

Finally, the remaining parameters of the RRR models (4)-(6) and (8) are then estimated by OLS after fixing the various matrices $\psi$ 's to their estimated values.

\section{Simultaneously modelling differing forms of common features}

A serious limitation of the existing methods for common features analysis is that they cannot handle the possible coexistence of differing types of reduced-rank restrictions in the same VECM. Consider, for instance, the following model:

$$
\Delta y_{t}=\Phi_{0}+\delta_{A \perp} \psi_{A}^{\prime} \Delta y_{t-1}+\delta_{B \perp} \psi_{B}^{\prime} \beta_{*}^{\prime} y_{t-1}^{*}+\delta_{F \perp} \psi_{F}^{\prime}\left(\Delta y_{t-2}^{\prime}, \ldots, \Delta y_{t-p+1}^{\prime}\right)^{\prime}+\varepsilon_{t},
$$

where $\delta_{F}=\left(\delta_{A}, \delta_{B}\right), \delta_{A}$ is an $n \times s_{1}$ matrix, $\delta_{B}$ is an $n \times s_{2}$ matrix, the rank of matrix $\delta_{F}$ equals $\left(s_{1}+s_{2}\right)$, and $\psi_{A}$ and $\psi_{B}$ are, respectively, $r \times\left(n-s_{1}\right)$ and $n \times\left(n-s_{2}\right)$ matrices with full column ranks. Hence, model (11) exhibit both $s_{1}$ WFs and $s_{2}$ PSCCFs.

Assume now that series $\Delta y_{t}$ are instead generated by the model below

$$
\Delta y_{t}=\Phi_{0}+\delta_{C \perp} \psi_{C}^{\prime}\left(\Delta y_{t-1}^{\prime}, y_{t-1}^{* \prime} \beta_{*}\right)^{\prime}+\delta_{F \perp} \psi_{F}^{\prime}\left(\Delta y_{t-2}^{\prime}, \ldots, \Delta y_{t-p+1}^{\prime}\right)^{\prime}+\varepsilon_{t},
$$

where $\delta_{C}=\delta_{F} \omega, \omega$ is a full-rank $s \times s_{1}$ matrix, and $\psi_{C}$ is an $(n+r) \times\left(n-s_{1}\right)$ matrix with full column rank. It is clear that $s_{1}$ out of the $s$ WFPs of model (12) are indeed SCCFs. 
Table 3

Tests for overidentifying restrictions in the WFP model

\begin{tabular}{lll}
\hline Model & $H^{\prime}$ s matrices & $d_{2}$ \\
\hline$(4)$ & $H_{1}=\left(I_{n}, 0_{n \times(n+r)}\right)^{\prime}$ & $s \times(n+r)$ \\
$(6)$ & $H_{2}=\left(I_{n+r}, 0_{(n+r) \times n}\right)^{\prime}$ & $s \times n$ \\
$(5)$ & $H_{3}=\left(\begin{array}{ccc}I_{n} & 0_{n \times r} & 0_{n \times n} \\
0_{n \times n} & 0_{n \times r} & I_{n}\end{array}\right)$ & $s \times r$ \\
\hline
\end{tabular}

Even if the presence of these differing forms of common features can be tested by means of the statistic (10), it is not possible to impose the implied reduced-rank structure on the estimated model. In this section we try to overcome such a limitation. Based on Cubadda (2007), one can use the following RRR model:

$$
u_{t}=\widetilde{\Phi}_{0}+\delta_{\perp} \Psi^{\prime} w_{t-1}+\widetilde{\varepsilon}_{t},
$$

where $u_{t}=\left(\Delta y_{t}^{\prime}, y_{t-1}^{* \prime} \beta_{*}, \Delta y_{t-1}^{\prime}\right)^{\prime}, \widetilde{\Phi}_{0}=\left(\Phi_{0}^{\prime}, 0_{1 \times(r+n)}\right)^{\prime}, \widetilde{\varepsilon}_{t}=\left(\varepsilon_{t}^{\prime}, 0_{1 \times(r+n)}\right)^{\prime}, \delta$ is an $(2 n+r) \times s$ matrix with $s<n$, and $\Psi$ is an $(r+p n-n) \times(2 n+r-s)$ matrix such that

$$
\delta_{\perp} \Psi^{\prime}=\left(\begin{array}{cc}
\left(\alpha, \Gamma_{1}\right) & \left(\Gamma_{2}, \ldots, \Gamma_{p-1}\right) \\
I_{r+n} & 0_{(r+n) \times(p n-2 n)}
\end{array}\right) .
$$

Since model (13) is an isomorphic representation of model (8), statistical inference based on the solution of

$$
\text { CanCor }\left\{u_{t}, w_{t-1} \mid 1\right\}
$$

is identical to that for the existence of $s$ WFPs. However, since the other forms of common features are nested in model (13), inference on all of them can be conducted by means of a restricted solution of the canonical correlation problem (14).

Remark 3. Given that the terms $\left(y_{t-1}^{* \prime} \beta_{*}, \Delta y_{t-1}^{\prime}\right)^{\prime}$ are present in both $u_{t}$ and $w_{t-1}$, the $(n+r)$ largest canonical correlation coming from (13) are exactly equal to one. However, the $n$ smallest of such canonical correlations are the same as those required for statistical inference on model (8) in Table 1.

In a manner similar to that of Johansen (1996), let us consider linear restrictions of the form $\delta=H \theta$, where $H$ is a known $(2 n+r) \times g$ matrix with full column rank, and $\theta$ is a $g \times s$ matrix to be estimated. Let $\widehat{v}_{i}$ denotes the $i$ th smallest squared canonical correlation, and $\widehat{\varphi}_{i}^{H^{\prime} u}$ denote the associated canonical coefficients of $H^{\prime} u_{t}$ drawn from the following canonical correlation program:

$$
\text { CanCor }\left\{H^{\prime} u_{t}, w_{t-1} \mid 1\right\} \text {. }
$$

Then the LR test statistic for the null hypothesis $\delta=H \theta$ is given by

$$
L R_{2}=T \sum_{i=1}^{s} \ln \left(\frac{1-\widehat{\omega}_{i}}{1-\widehat{v}_{i}}\right), \quad s=1, \ldots, n,
$$

where $\widehat{\omega}_{i}$ denotes the $i$ th smallest squared canonical correlation drawn from the solution of (14), and the estimates of the parameters $\theta$ are given by $\left[\widehat{\varphi}_{1}^{H^{\prime} u}, \ldots, \widehat{\varphi}_{s}^{H^{\prime} u}\right]$. Under the null hypothesis the test statistic $(16)$ has a $\chi^{2}\left(d_{2}\right) \operatorname{limit}$ distribution, where $d_{2}=s(2 n+r-g)$.

Let us suppose that $s$ WFPs exist and one wishes to test if a more restricted form of common features exists in the data. For this purpose, it is required to solve the restricted canonical correlation program (15) for proper choices of the matrix $H$ and to use the test statistic (16) as detailed in Table 3.

When differing forms of common features are simultaneously present in the data, a more elaborated statistical approach is called for. For the sake of simplicity, the focus is only on the case where two differing types of common features coexist; but the proposed methods can be easily generalized. It is convenient to separate the treatment of the case where PSCCF's and WF's are both present as in model (11) from that of the case of nested common features structures, which occurs in model (12). 


\subsection{Coexistence of PSCCFs and WFs}

Let us start by reparametrizing model (11) in terms of model (13). In view of Table 3, this is obtained by writing $\delta=\left(\delta_{2}, \delta_{3}\right)$ where $\delta_{2}=H_{2} \theta_{2}$ and $\delta_{3}=H_{3} \theta_{3}$. Hence, premultiplying both sides of model (13) by, respectively, $H_{2}^{\prime}$ and $H_{3}^{\prime}$ yields

$$
H_{2}^{\prime} u_{t}=H_{2}^{\prime} \widetilde{\Phi}_{0}+H_{2}^{\prime} \delta_{\perp} \Psi^{\prime} w_{t-1}+H_{2}^{\prime} \widetilde{\varepsilon}_{t},
$$

and

$$
H_{3}^{\prime} u_{t}=H_{3}^{\prime} \widetilde{\Phi}_{0}+H_{3}^{\prime} \delta_{\perp} \Psi^{\prime} w_{t-1}+H_{3}^{\prime} \widetilde{\varepsilon}_{t} .
$$

By taking, respectively, the expectation of $H_{2}^{\prime} u_{t}$ conditional to $\delta_{3}^{\prime} u_{t}$ and that of $H_{3}^{\prime} u_{t}$ conditional to $\delta_{2}^{\prime} u_{t}$ one obtains

$$
\begin{aligned}
H_{2}^{\prime} u_{t} & =H_{2}^{\prime} \widetilde{\Phi}_{0}+H_{2}^{\prime} \delta_{\perp} \Psi^{\prime} w_{t-1}+\mathrm{E}\left(H_{2}^{\prime} \widetilde{\varepsilon}_{t} \mid \delta_{3}^{\prime} u_{t}\right)+\xi_{2, t} \\
& \equiv \mu_{2}+H_{2}^{\prime} \delta_{\perp} \Psi^{\prime} w_{t-1}+\gamma_{2} \delta_{3}^{\prime} u_{t}+\xi_{2, t},
\end{aligned}
$$

and

$$
\begin{aligned}
H_{3}^{\prime} u_{t} & =H_{3}^{\prime} \widetilde{\Phi}_{0}+H_{3}^{\prime} \delta_{\perp} \Psi^{\prime} w_{t-1}+\mathrm{E}\left(H_{3}^{\prime} \widetilde{\varepsilon}_{t} \mid \delta_{2}^{\prime} u_{t}\right)+\xi_{3, t} \\
& \equiv \mu_{3}+H_{3}^{\prime} \delta_{\perp} \Psi^{\prime} w_{t-1}+\gamma_{3} \delta_{2}^{\prime} u_{t}+\xi_{3, t},
\end{aligned}
$$

where $\xi_{2, t}$ and $\xi_{3, t}$ are i.i.d. Gaussian innovations with respect to $\Omega_{t-1}$.

In view of the above partial RRR models, ML inference on the parameters $\theta_{3}$ for fixed $\delta_{2}$ is obtained by solving

$$
\text { CanCor }\left\{H_{3}^{\prime} u_{t}, w_{t-1} \mid\left(1, u_{t}^{\prime} \delta_{2}\right)^{\prime}\right\},
$$

and, vice versa, ML inference on $\theta_{2}$ having fixed $\delta_{3}$ is obtained by the solution of

$$
\text { CanCor }\left\{H_{2}^{\prime} u_{t}, w_{t-1} \mid\left(1, u_{t}^{\prime} \delta_{3}\right)^{\prime}\right\} .
$$

Hence, the likelihood function of $\delta$ can be maximized by a linear switching algorithm similar to that proposed for cointegration analysis by Johansen and Juselius (1992), Johansen (1996), and Paruolo (2006b, c). This algorithm, which increases the likelihood function in each step, proceeds as follows:

(1) Estimate $\delta$ unrestricted and obtain an initial estimate of $\delta_{2}$ as the orthogonal projection of $\delta$ onto $H_{2}$. This is obtained as $\widehat{\delta}_{2}=H_{2}\left(H_{2}^{\prime} H_{2}\right)^{-1} H_{2}^{\prime} \widehat{\delta}$. Alternative choices of the starting values are discussed in details in Paruolo (2006c).

(2) For fixed $\delta_{2}=\widehat{\delta}_{2}$, obtain $\widehat{\delta}_{3}=H_{3} \widehat{\theta}_{3}$, where $\widehat{\theta}_{3}$ are the canonical coefficients of $H_{3}^{\prime} u_{t}$ associated with the $s_{2}$ smallest eigenvalues drawn from the solution of (17).

(3) For fixed $\delta_{3}=\widehat{\delta}_{3}$, obtain $\widehat{\delta}_{2}=H_{2} \widehat{\theta}_{2}$, where $\widehat{\theta}_{3}$ are the canonical coefficients of $H_{2}^{\prime} u_{t}$ associated with the $s_{1}$ smallest eigenvalues drawn from the solution of (18).

(4) Repeat (2) and (3) until numerical convergence occurs.

The LR test statistic for the null hypothesis $\delta=\left(H_{2} \theta_{2}, H_{3} \theta_{3}\right)$ versus the alternative that $\delta$ is unrestricted is then given by

$$
L R_{3}=T \log \left(\operatorname{det}\left(\widehat{\Sigma}_{\varepsilon}\right) \operatorname{det}\left(\widetilde{\Sigma}_{\varepsilon}\right)^{-1}\right),
$$

where $\widehat{\Sigma}_{\varepsilon}$ and $\widetilde{\Sigma}_{\varepsilon}$ are the residual covariance matrices of models (8) and (11), respectively. The test statistic (19) follows asymptotically a $\chi^{2}\left(d_{3}\right)$ distribution, where $d_{3}=\left(s_{1} n+s_{2} r-s\right)$. Notice that Paruolo (2006b) corrected a common error in previous formulations of such a LR test statistic.

Regarding the estimators of the parameters of model (11), $\widehat{\psi}_{A}$ is given by the canonical coefficients of $\Delta y_{t-1}$ associated with the $\left(n-s_{1}\right)$ largest canonical correlations drawn from (17), $\widehat{\psi}_{B}$ is given by the canonical coefficients of $\beta_{*}^{\prime} y_{t-1}^{*}$ associated with the $\left(n-s_{2}\right)$ largest canonical correlations drawn from (18), and $\widehat{\psi}_{F}$ is obtained by regressing $\left(\widehat{\delta}_{F \perp}^{\prime} \widehat{\delta}_{F \perp}\right)^{-1} \widehat{\delta}_{F \perp}^{\prime} r_{0 t}$ on $r_{1 t}$, where $\widehat{\delta}_{F}$ is given by the first $n$ rows of the matrix $\left(\widehat{\delta}_{2}, \widehat{\delta}_{3}\right)$, and $r_{0 t}$ and $r_{1 t}$ are, respectively, 
the residuals of a regression of $\Delta y_{t}$ and $\left(\Delta y_{t-2}^{\prime}, \ldots, \Delta y_{t-p+1}^{\prime}\right)^{\prime}$ on $\left(\Delta y_{t-1}^{\prime} \widehat{\psi}_{A}, y_{t-1}^{* \prime} \beta_{*} \widehat{\psi}_{B}\right)^{\prime}$. Finally, the remaining parameters of model (11) are estimated by OLS after fixing the parameter matrices $\psi_{A}, \psi_{B}$ and $\psi_{F}$ to their estimated values.

Remark 4. Notice that the necessary and sufficient condition for identification of the parameters $\left(\theta_{2}^{\prime}, \theta_{3}^{\prime}\right)$ (see Johansen, $1995)$ is here satisfied $\operatorname{since} \operatorname{rank}\left(H_{2 \perp}^{\prime} H_{3}\right)=\operatorname{rank}\left(H_{3 \perp}^{\prime} H_{2}\right)=n \geqslant s$.

\subsection{Nested forms of common features}

In order to simplify notation, let us suppose that the statistical problem consists of testing whether $s_{1}$ out of the $s$ WFPs are indeed common features of a restricted form. However, it will be clear that the proposed solution applies to any case of nested common features. Hence, let us write $\delta=\left(\delta_{r}, \delta_{u}\right)$, where $\delta_{r}=H_{j} \theta_{j}$ for $j=1,2,3, \theta_{j}$ is a $g_{j} \times s_{1}$ matrix with full column rank, and $\delta_{u}$ is an $n \times s_{2}$ matrix with full column rank.

A reasoning which is similar to that of the previous subsection yields to the following equations:

$$
\begin{aligned}
H_{j} u_{t} & =H_{2}^{\prime} \widetilde{\Phi}_{0}+H_{j}^{\prime} \delta_{\perp} \Psi^{\prime} w_{t-1}+\mathrm{E}\left(H_{j}^{\prime} \widetilde{\varepsilon}_{t} \mid \delta_{u}^{\prime} u_{t}\right)+\xi_{r, t} \\
& \equiv \mu_{r}+H_{j}^{\prime} \delta_{\perp} \Psi^{\prime} w_{t-1}+\gamma_{r} \delta_{u}^{\prime} u_{t}+\xi_{r, t},
\end{aligned}
$$

and

$$
\begin{aligned}
H_{j \perp}^{\prime} u_{t} & =H_{j \perp}^{\prime} \widetilde{\Phi}_{0}+H_{j \perp}^{\prime} \delta_{\perp} \Psi^{\prime} w_{t-1}+\mathrm{E}\left(H_{j \perp}^{\prime} \widetilde{\varepsilon}_{t} \mid \delta_{r}^{\prime} u_{t}\right)+\xi_{u, t} \\
& \equiv \mu_{u}+H_{j \perp}^{\prime} \delta_{\perp} \Psi^{\prime} w_{t-1}+\gamma_{u} \delta_{r}^{\prime} u_{t}+\xi_{u, t},
\end{aligned}
$$

where $\xi_{r, t}$ and $\xi_{u, t}$ are i.i.d. Gaussian innovations with respect to $\Omega_{t-1}$. Hence, the statistical problem is solved by the following switching algorithm:

I. Estimate $\delta$ unrestricted and obtain an initial estimate of $\delta_{r}$ as $\widehat{\delta}_{r}=H_{j}\left(H_{j}^{\prime} H_{j}\right)^{-1} H_{j}^{\prime} \widehat{\delta}$.

II. For fixed $\delta_{r}=\widehat{\delta}_{r}$, obtain $\widehat{\delta}_{u}=H_{j \perp} \widehat{\theta}_{u}$, where $\widehat{\theta}_{u}$ are the canonical coefficients of $H_{j \perp}^{\prime} u_{t}$ associated with the $s_{2}$ smallest eigenvalues drawn from the solution of

$$
\operatorname{CanCor}\left\{H_{j \perp}^{\prime} u_{t}, w_{t-1} \mid\left(1, u_{t}^{\prime} \delta_{r}\right)^{\prime}\right\} \text {. }
$$

Notice that $\widehat{\delta}_{u}$ is restricted to $H_{j \perp}^{\prime}$ in order to avoid a singularity problem in the canonical correlation problem (20).

III. For fixed $\delta_{u}=\widehat{\delta}_{u}$, obtain $\widehat{\delta}_{r}=H_{j} \widehat{\theta}_{j}$, where $\widehat{\theta}_{j}$ as the canonical coefficients of $H_{j}^{\prime} u_{t}$ associated with the $s_{1}$ smallest eigenvalues drawn from the solution of

$$
\operatorname{CanCor}\left\{H_{j}^{\prime} u_{t}, w_{t-1} \mid\left(1, u_{t}^{\prime} \delta_{u}\right)^{\prime}\right\} .
$$

IV. Repeat II and III until numerical convergence occurs.

The LR test statistic for the null hypothesis $\delta_{r}=H_{j} \theta_{j}$ versus the alternative that $\delta_{r}$ is unrestricted is again given by (19), where $\widetilde{\Sigma}_{\varepsilon}$ is in this case the residual covariance matrix of the model associated with matrix $H_{j}$ in Table 3 , and $d_{3}=s_{1}\left(d_{2} / s-1\right)$, see again Table 3 .

Regarding the estimators of the RRR parameters, let us focus on model (12), i.e., $j=1$. Then $\widehat{\psi}_{C}$ is given by the canonical coefficients of $\left(\Delta y_{t-1}^{\prime}, y_{t-1}^{* \prime} \beta_{*}\right)^{\prime}$ associated with the $\left(n-s_{1}\right)$ largest canonical correlations drawn from (20), and $\widehat{\psi}_{F}$ is obtained by regressing $\left(\widehat{\delta}_{F \perp}^{\prime} \widehat{\delta}_{F \perp}\right)^{-1} \widehat{\delta}_{F \perp}^{\prime} r_{0 t}$ on $r_{1 t}$, where $\widehat{\delta}_{F}$ is given by the first $n$ rows of the matrix $\left(\widehat{\delta}_{r}, \widehat{\delta}_{u}\right)$, and $r_{0 t}$ and $r_{1 t}$ are, respectively, the residuals of a regression of $\Delta y_{t}$ and $\left(\Delta y_{t-2}^{\prime}, \ldots, \Delta y_{t-p+1}^{\prime}\right)^{\prime}$ on $\widehat{\psi}_{C}^{\prime}\left(\Delta y_{t-1}^{\prime}, y_{t-1}^{* \prime} \beta_{*}\right)^{\prime}$. The other coefficient matrices of models (12) are then estimated by OLS after fixing the parameter matrices $\psi_{C}$ and $\psi_{F}$ to their estimated values. By a similar reasoning, one obtains the estimators of the RRR parameters when $j=2,3$. 


\section{Empirical example: common features of the US business cycle indicators}

In order to illustrate the practical value of the proposed methods, let us consider the monthly indicators that The Conference Board uses to build the composite coincident indicator of the business cycle in the US. In particular, the empirical analysis concerns the logarithms of employees on non-agricultural payrolls, personal income less transfer payments (corrected for additive outliers corresponding to 1992.12 and 1993.12), industrial production, and manufacturing and trade sales for the period 1974.1-2003.7. These series are graphed in Fig. 1. Although the data are available from 1959.1, only the post first oil-shock period is used because a preliminary application of the test by Bai et al. (1998) revealed that a VAR model of these series is affected by a structural break occurring in the late 1973.

According to the longest significant lag rule, a VAR(6) with a linear trend is fitted to the data. This model seems to reproduce successfully the dynamic features of the data since the null hypothesis of no residuals autocorrelation is clearly not rejected. Table 4 reports the results of the Johansen's LR tests for cointegration, which suggest the existence of one cointegrating vector.

Having fixed $r=1$, the presence of the various forms of common cyclical features is scrutinized. The results, reported in Table 5, indicate $s=1$ for the SCCF, WF and PSCCF, and $s=2$ for the WFP. Overall, the evidence favours the existence of one unrestricted WFP, and one common feature of a restricted form.

Since the presence of two unrestricted WFPs and one cointegration vector implies that one PSCCF exists, it is of interest to check whether the restricted form of common feature is either a WF or an SCCF. A test for the former

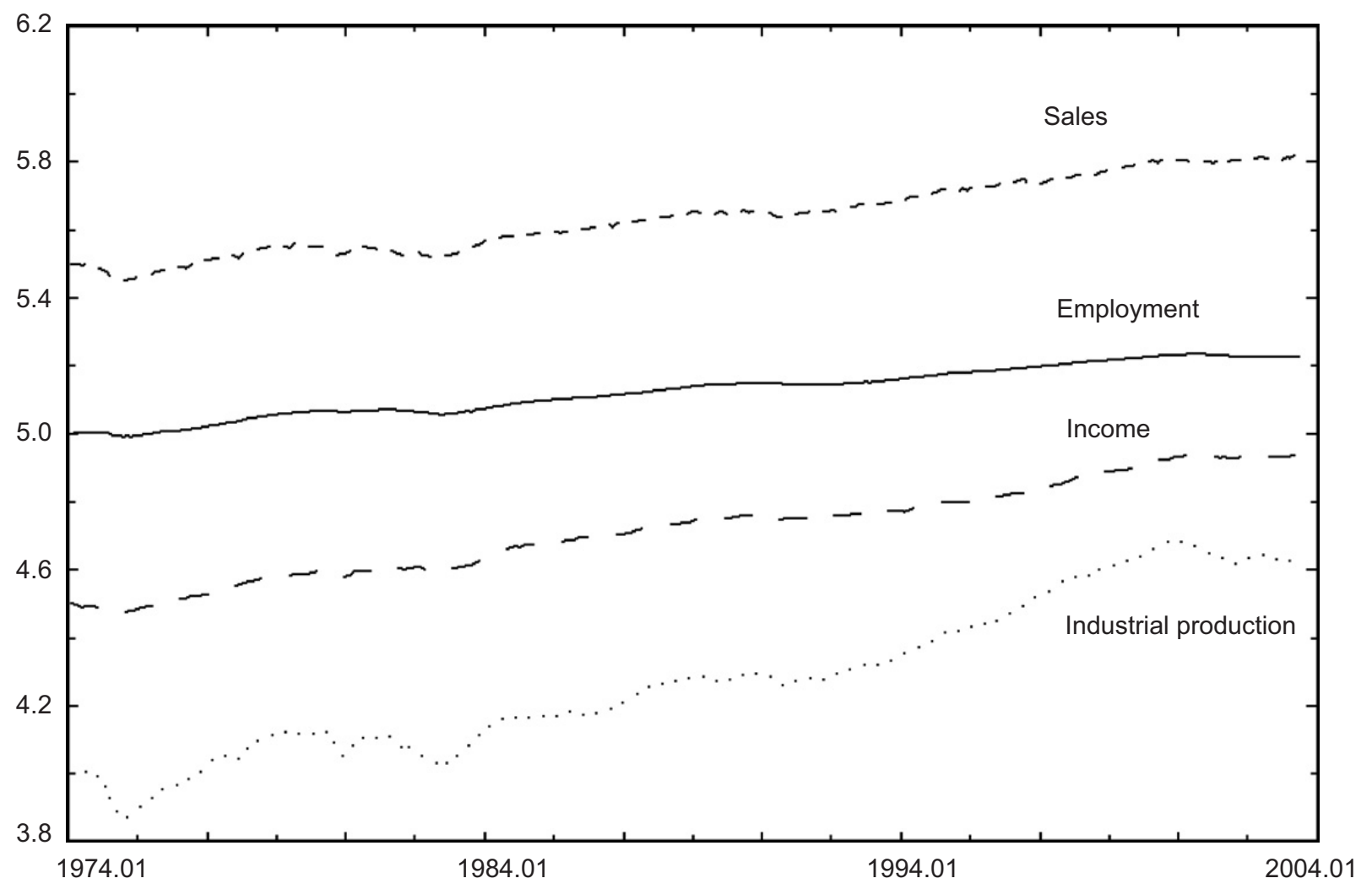

Fig. 1. The US business cycle coincident indicators.

Table 4

Trace tests for cointegration

\begin{tabular}{llll}
\hline$r=0$ & $r \leqslant 1$ & $r \leqslant 2$ & $r \leqslant 3$ \\
\hline $72.33^{*}$ & 38.26 & 21.20 & 9.236 \\
\hline
\end{tabular}

*Significant at the $10 \%$ confidence level. 
Table 5

Common features tests

\begin{tabular}{lllr}
\hline & $s \geqslant 1$ & $s \geqslant 2$ & $s \geqslant 3$ \\
\hline SCCF & $20.20^{*}$ & 88.70 & 187.8 \\
WF & $17.14^{*}$ & 60.24 & 495.7 \\
PSCCF & $16.25^{*}$ & 59.74 & 421.1 \\
WFP & $15.56^{*}$ & $36.16^{*}$ & 118.4 \\
\end{tabular}

* Significant at the $10 \%$ confidence level.

Table 6

Estimates of the common features' relationships

\begin{tabular}{ll}
\hline SCCF & $(1,-1.571,0.423,0.038)^{\prime} \Delta y_{t}$ \\
WFP & $(1,0.445,-0.668,-0.469)^{\prime} \Delta y_{t}-0.004 \widehat{\beta}_{*}^{\prime} y_{t-1}^{*}+(0.673,0.191,0.031,-0.100)^{\prime} \Delta y_{t-1}$
\end{tabular}

hypothesis produces a test statistic equal to 7.64 with a $p$-value equal to 0.27 , whereas a test for the latter hypothesis produces a test statistic equal to 8.65 with a $p$-value equal to 0.28 . Since the SCCF is nested within the WF, these results put forward the coexistence of one unrestricted WFP and one SCCF. Notice that switching algorithm was terminated when the relative decrease of $\operatorname{det}\left(\widetilde{\Sigma}_{\varepsilon}\right)$ become inferior than $0.01 \%$. Overall, seven iterations were needed for numerical convergence. The estimates of the associated common feature vectors are reported in Table 6.

Remarkably, the model that incorporates the above common features' relationships has 52 parameters, whereas the model that only satisfies the SCCF restrictions calls for the estimation of 74 parameters.

\section{Conclusions}

This paper offers an approach for simultaneously modelling differing forms of common cyclical features among I(1) time series. After showing that several existing forms of common features are nested within a new model, namely the weak form of PSCCFs, some iterative procedures are proposed for testing and imposing diverse forms of common features upon a cointegrated VAR model. The empirical application reveals that the new methods provide a model of the US business cycle indicators that is considerably more parsimonious than those obtained using the pre-existing concepts of common features.

\section{Acknowledgements}

Previous drafts of this paper were presented at the third IASC World Conference on Computational Statistics \& Data Analysis in Limassol, and 61st European Meeting of the Econometric Society in Vienna. I wish to thank three anonymous referees and the associate editor Stephen Pollock, as well as Bertrand Candelon, Alain Hecq and Paolo Paruolo, for useful comments and corrections. Financial support from MIUR is gratefully acknowledged. The usual disclaimers apply.

\section{References}

Ahn, S.K., Reinsel, G.C., 1988. Nested reduced-rank autoregressive models for multiple time series. J. Amer. Statist. Assoc. 13, $352-375$. Anderson, T.W., 2002. Canonical correlation analysis and reduced rank regression in autoregressive models. Ann. Statist. 30, $1134-1154$. Bai, J., Lumsdaine, R.L., Stock, J.H., 1998. Testing for and dating breaks in multivariate time series. Rev. Econom. Stud. 65, $395-432$.

Beveridge, S., Nelson, C.R., 1981. A new approach to decomposition of economic time series into permanent and transitory components with particular attention to measurement of the business cycle. J. Monetary Econom. 7, 151-174.

Cubadda, G., 1999. Common cycles in seasonal non-stationary time series. J. Appl. Econometrics 14, $273-291$.

Cubadda, G., 2007. A reduced rank regression approach to coincident and leading indexes building. Oxford Bull. Econom. Statist. 69, $271-292$.

Cubadda, G., Hecq, A., 2001. On non-contemporaneous short-run comovements. Econom. Lett. 73, 389-397.

Cubadda, G., Omtzigt, P., 2005. Small-sample improvements in the statistical analysis of seasonally cointegrated systems. Comput. Statist. Data Anal. 49, 333-348.

Engle, R.F., Granger, C.W.J., 1987. Cointegration and error correction: representation, estimation and testing. Econometrica 55, $251-278$. 
Engle, R.F., Kozicki, S., 1993. Testing for common features (with comments). J. Business Econom. Statist. 11, 369-395.

Ericsson, N., 1993. Comment (on Engle and Kozicki, 1993). J. Business Econom. Statist. 11, 380-383.

Haldrup, N., Hylleberg, S., Pons, G., Sansó, A., 2007. Common periodic correlation features and the interaction of stocks and flows in daily airport data. J. Business Econom. Statist. 25, 21-32.

Hecq, A., Palm, F.C., Urbain, J.P., 2000. Permanent-transitory decomposition in VAR models with cointegration and common cycles. Oxford Bull. Econom. Statist. 62, 511-532.

Hecq, A., Palm, F.C., Urbain, J.P., 2006. Common cyclical features analysis in VAR Models with cointegration. J. Econometrics 132, $117-141$.

Johansen, S., 1995. Identifying restrictions of linear equations with application to simultaneous equations and cointegration. J. Econometrics 69, $111-132$.

Johansen, S., 1996. Likelihood-Based Inference in Cointegrated Vector Autoregressive Models. Oxford University Press, Oxford.

Johansen, S., Juselius, K., 1992. Testing structural hypotheses in a multivariate cointegration analysis of the PPP and UIP for UK. J. Econometrics 53, 211-244.

Lucas, R., 1977. Understanding business cycles. Carnegie-Rochester Seri. Public Pol. 5, 7-29.

Mizon, G.E., 1995. A simple message for autocorrelation correctors: don't. J. Econometrics 69, 267-288.

Paruolo, P., 2003. Common dynamics in I(1) systems. Department of Economics, University of Insubria WP, 35.

Paruolo, P., 2006a. Common trends and cycles in I(2) VAR systems. J. Econometrics 132, 143-168.

Paruolo, P., 2006b. The likelihood ratio test for the rank of a cointegration submatrix. Oxford Bull. Econom. Statist. 68, 921-948.

Paruolo, P., 2006c. Finite sample comparison of alternative tests on the rank of a cointegration submatrix. Department of Economics, University of Insubria WP, 6.

Proietti, T., 1997. Short-run dynamics in cointegrated systems. Oxford Bull. Econom. Statist. 59, 405-422.

Reinsel, G.C., Velu, R.P., 1998. Multivariate Reduced-Rank Regression: Theory and Applications. Lecture Notes in Statistics. Springer, New York. Sargan, J.D., 1983. Identification in models with autoregressive errors. In: Karlin, S., Amemiya, T., Goodman, L.A. (Eds.), Studies in Econometrics, Time Series and Multivariate Statistics. Academic Press, New York, pp. 169-205.

Vahid, F., Engle, R.F., 1993. Common trends and common cycles. J. Appl. Econometrics 8, 341-360. 